\title{
Investigation of $\alpha$-Ti plastic anisotropy under thermomechanical loadings: temperature influence on deformation mechanisms
}

\author{
K. E. Agbovi ${ }^{1}{ }^{*}$, J. Fajoui ${ }^{1}$, B. Girault ${ }^{1}$, D. Gloaguen ${ }^{1}$, S. Kabra ${ }^{2}$, J. Kelleher ${ }^{2}$, W. Kockelmann ${ }^{2}$
}

${ }^{1}$ Institut de Recherche en Génie Civil et Mécanique, GeM (UMR CNRS 6183), Université de Nantes, 58, rue Michel Ange - BP 420,44606 Saint-Nazaire Cedex, France

2 ISIS Neutron and Muon Source, Science and Technology Facilities Council, Rutherford Appleton Laboratory, Didcot, OX11 0QX, United Kingdom

${ }^{*}$ Corresponding author: kodjo.agbovi@etu.univ-nantes.fr

$\underline{\text { Abstract }}$

In situ neutron diffraction method has been used to investigate deformation mechanisms (slip and twinning deformation modes) during uniaxial tensile tests of extruded atitanium alloy at room temperature, 100 and $300{ }^{\circ} \mathrm{C}$. For this purpose, the prediction of an Elasto-Plastic Self-Consistent (EPSC) model was compared with the experimental data. Results show that there is a good agreement between the simulations and neutron diffraction measurements. The EPSC model enables realistic predictions of not only the elastic lattice strains developed in variously oriented grain families but also the macroscopic stress-strain response within the bulk material.

\section{Introduction}

Titanium (Ti) alloys are important for biomedical applications and are also widely used in the aviation industry [1,2]. This is due to their high specific strength and good corrosion resistance at elevated temperatures. Metallic materials such as titanium alloys undergo thermomechanical loadings that can affect their mechanical performances and lifetime during their manufacturing or when they are subjected to different loads and thermal conditions. A deeper knowledge of the mechanical behavior of these heterogeneous materials and their deformation sensitivity, in particular as regard to temperature, is thus essential to master the shaping methods and the service life of titanium alloys.

At room temperature, it is well established that prismatic slip $\{10.0\}(11.0\}$ is the easiest deformation mode to be activated in $\alpha$-Ti. Pyramidal $>$ slip $\{10.1\}(11.3)$, pyramidal $>\operatorname{slip}\{10.1\}(11.0)$ and basal slip $\{00.2\}(11.0\}$ are generally presented as secondary slipping modes [3]. In particular, $\{10.1\}(11.3)$ slip produces plastic deformation with a component out of the basal plane (along the c-axis). Deformation along c-axis can also be accommodated by twinning on the $\{10.2\}$ (10. $\overline{1}\rangle$ or $\{11.2\}\langle 11 . \overline{3}\rangle$ systems [3-6]. Information on the activity of slip systems, the role of twinning deformation, grain reorientation, especially as regard to high temperatures, are thus key elements to understand the mechanical behavior of these alloys and to optimize their mechanical properties.

There is some disagreement within the literature regarding the exact deformation mechanisms during plastic deformation of titanium alloys, especially at high temperatures [7,21-23]. Up to now, it remains indeed unclear which deformation systems are actually activated in a polycrystal during straining and how the temperature affects the activation sequence. The main objective of this study is to investigate the evolution of the internal strain under thermomechanical loading conditions. Neutron diffraction experiments provide a comprehensive data set for testing an Elasto-Plastic Self-Consistent model that integrates the influence of temperature, which is developed to predict macroscopic mechanical behavior and to interpret the lattice strain development obtained in neutron diffraction.

\section{Experimental details}

2.1 Material

The investigated material is a commercially pure $\alpha$-titanium. Its chemical composition is $\mathrm{C}(0.012), \mathrm{Fe}(0.164), \mathrm{N}(0.0078), \mathrm{O}(0.137), \mathrm{H}(0.001)$ and balance Ti (wt.\%). The material was received in an extruded bar form. Tensile sample (gage dimensions: diameter $\varnothing=8 \mathrm{~mm}$, length $1_{0}=42 \mathrm{~mm}$ ) for the in situ neutron diffraction experiments was machined along the extrusion direction from the as-received rod. The metallographic characterization has revealed an average grain size of approximately $50 \mu \mathrm{m}$. Texture measurement were performed on the GEM beamline at the ISIS neutron spallation source [8]. Measurements of the initial texture (Fig.1) revealed that the samples have a strong asymmetric texture in the ND-TD (ND: Normal Direction, TD: Transverse Direction) plane, perpendicular to the loading axis (LD). The Orientation Distribution Function (ODF) calculation has been performed with experimental Pole Figures (PF) $\{10.0\},\{00.2\},\{10.1\},\{11.0\}$ and $\{10.2\}$ with help of WIMV 
algorithm implemented in the BEARTEX program package [8]. The measured initial texture was used as an input to the EPSC model: the polycrystal is then represented by a weighted discrete distribution of orientations.

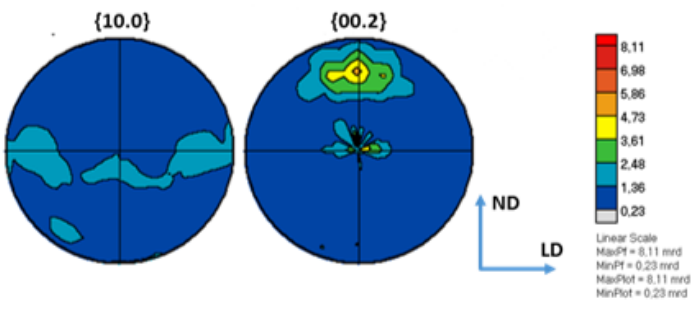

Fig. 1: Initial crystallographic texture of the studied sample

2.2 Neutron diffraction measurements

Tensile tests were performed at ambient, 100 and $300{ }^{\circ} \mathrm{C}$ using the ENGIN-X neutron diffractometer at the ISIS spallation neutron source (Didcot, Oxforshire, UK). The measurement steps were controlled by a $50 \mathrm{kN}$ Instron testing machine with a strain rate of $10^{-5} \mathrm{~s}^{-1}$. The incident slits and radial collimators defined a scattering volume of $4 \times 4 \times 4 \mathrm{~mm}^{3}$ with counting times of 43 minutes per diffraction measurement. The macroscopic strain was determined using an extensometer that spanned the irradiated region. A K-thermocouple was used to monitor and control the sample temperature.

To avoid any strain relaxation due to the creep behavior at room temperature in the plastic regime, samples were first deformed to the desired macroscopic strain, and then immediately unloaded, studying just the residual strains resulting from a given increment of plastic deformation, at zero applied load. The ENGIN-X instrument presents a horizontal loading axis positioned at $45^{\circ}$ to the incident beam. Two detector banks are set up horizontally at $\pm 90^{\circ}$ from the incident beam, enabling simultaneous measurements of lattice strains from plane families lying parallel (along loading direction, LD) and perpendicular (along transverse direction, TD) directions as regard to the applied load (Fig. 2). The Time-Of-Flight (TOF) technique enables collecting simultaneously an entire diffraction pattern (effective d-spacing range from 0.88 to 2.63 $\AA$ ) on each detector, owing to the specificity of the neutron beam. The detector banks cover $\pm 14^{\circ}$ in the horizontal plane and $\pm 21^{\circ}$ in the vertical plane [10].

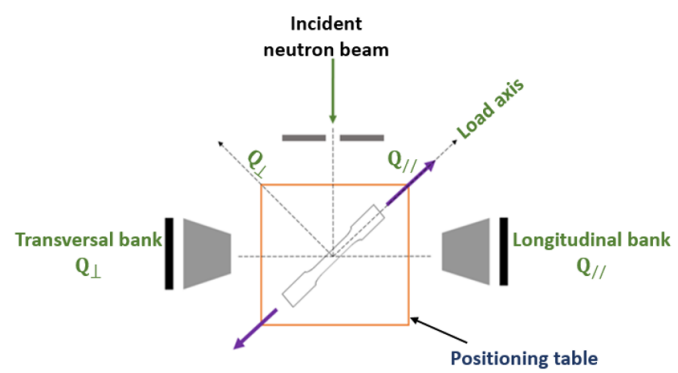

Fig. 2: Schematic view of the set-up used on the neutron diffraction instrument ENGIN-X

Single peak fitting was performed using the Open Genie code [11] thanks to Gaussian mathematical function. The lattice strain $(\varepsilon(h k . /))$ va of a grain group having common \{hk.1\} plane-normal, parallel to the diffraction vector Q (Fig. 2) i.e. grains fulfilling diffraction conditions can be calculated by the following formula:

$$
\left(\varepsilon(h k . I)_{\text {vd }}=\left((k(h k . I))_{\text {vd }}-d_{0}(h k . I)\right) / d_{0}(h k . I)\right.
$$

The reference point for this calculation is the initial measured $d_{a}(h k . I)$ for each lattice plane before deformation of the material. Ova stands for the averaging over diffracting grains for the considered $\left\{\right.$ hk.1\} reflection. $(d(h k . J))_{\text {va }}$ is the measured lattice spacing. Lattice strains presented in this work are given as micro-strain $(\mu \varepsilon$, units of $10^{-6}$ ). 


\subsection{Elasto-Plastic Self-Consistent (EPSC) modeling}

The EPSC approach employed in this work is based on the work of Kröner and Hill, described in [12-14]. In short, each grain is assumed to have the form of an ellipsoidal inclusion embedded in a homogeneous effective medium with anisotropic properties representative of the textured aggregate. In the original EPSC model, twinning is treated as a directional slip mechanism [12,13], the present study uses the "twinning scheme" which has been incorporated into the EPSC model by Clausen $e t$ al. [15]. This scheme accounts for: (i) the volume effect of twinning on texture evolution and, (ii) the stress relaxation associated with the twin formation. Hardening is taken into account in each deformation mode by the use of a linear law:

$$
i_{c}^{g}=\sum_{r} H^{5} \gamma^{r}
$$

where $\tau_{c}^{g}$ is the Critical Resolved Shear Stress (CRSS) rate and $\gamma^{r}$, the slip rate. $\mathscr{H}^{\sigma}$ are the hardening terms, relating the hardening of each system $g$ to the strain rate in the other systems $r$ and itself. In the present work, this matrix is simplified:

$$
H^{5}=a H^{\infty} \quad(3)
$$

$H^{\circ}$ is the self-hardening term and $H^{5}$ corresponds to the latent hardening (when $g^{1} r$ ). Due to lack of relevant data on latent hardening nature in titanium alloys, the interactions between different deformation modes are described by this very simple law. The $a$ factor defines the degree of latent hardening. The $a$ parameter is taken to be constant having a value of $a=1.3$ [12] for the simulations. The CRSS's ( $\left.\tau_{a}\right)$ and hardening parameters $\left(H^{p}\right)$ were used as fitting parameters to match the simulated response to the experimental macroscopic stress-strain curve and the elastic lattice strains measured for the individual grain orientations.

\section{Results}

The single crystal elastic constants used in this model are: $c_{11}=c_{22}=162.4 \mathrm{GPa}, c_{12}=92 \mathrm{GPa}, c_{13}=69 \mathrm{GPa}$ and $c_{14}=46.7 \mathrm{GPa}$ at room temperature $[16]$. To study the mechanical behavior at 100 and $300{ }^{\circ} \mathrm{C}$, the variation of $C_{l}$ with temperature has been taken into account. The elastic constants measured by Fisher et al. [17] are used. The temperature dependence of these constants can be described by a polynomial relation as proposed by Turner et al. [17]. The loading simulations begin with a discretized representation of the initial measured texture into 2002 distinct orientations. The assumed initial grain shape was spherical for the studied material. The Critical Resolved Shear Stress (CRSS) $\left(\mathrm{ra}_{\mathrm{a}}\right)$ and hardening parameters $\left(H^{\mathrm{p}}\right)$ of the deformation systems introduced in the calculations are summarized in Table 1. The CRSS

\begin{tabular}{|c|c|c|c|c|c|c|}
\hline \multirow{2}{*}{ Mechanisms } & \multicolumn{2}{|c|}{ Ambient } & \multicolumn{2}{|c|}{$100^{\circ} \mathrm{C}$} & \multicolumn{2}{|c|}{$300^{\circ} \mathrm{C}$} \\
\hline & $\mathrm{rg}$ & $H^{p o}$ & $r_{0}$ & $H^{p o}$ & $\mathrm{rg}$ & $H^{p o}$ \\
\hline Prismatic slip pr $>$ & 115 & 120 & 90 & 100 & 40 & 50 \\
\hline Basal slip bas > & 150 & 180 & 110 & 140 & 48 & 90 \\
\hline Pyramidal slip pyr > & 130 & 140 & 100 & 120 & 45 & 70 \\
\hline Pyramidal slip pyr > & 240 & 250 & 180 & 210 & 120 & 150 \\
\hline Tensile twin $\mathrm{ttw}-\{10.2\}$ & 290 & 280 & 230 & 240 & 165 & 200 \\
\hline Compression twin ctw- $\{11.2\}$ & 300 & 280 & 240 & 250 & 170 & 220 \\
\hline
\end{tabular}
values are in agreement with previous studies [18-20].

Table 1 : CRSS and hardening parameters (MPa) obtained with the model

True macroscopic stress-strain response of the $\alpha$-Ti alloy in tension along the extrusion direction were obtained at three different temperatures: ambient, 100 and $300^{\circ} \mathrm{C}$. The experimental and predicted results are shown in Fig. 3. They strongly depend on the temperature and plastic activity. Similar observations had been reported by other researchers [18]. As noted above, the plastic regime starting stress decreases with increasing temperature. For example, at $1.4 \%$ total deformation, stress values at room temperature, 100 and $300{ }^{\circ} \mathrm{C}$ are equal to 412,320 and $172 \mathrm{MPa}$, respectively. We can also notice the decrease of the experimental Young's modulus: $102 \pm 1$ GPa (room 


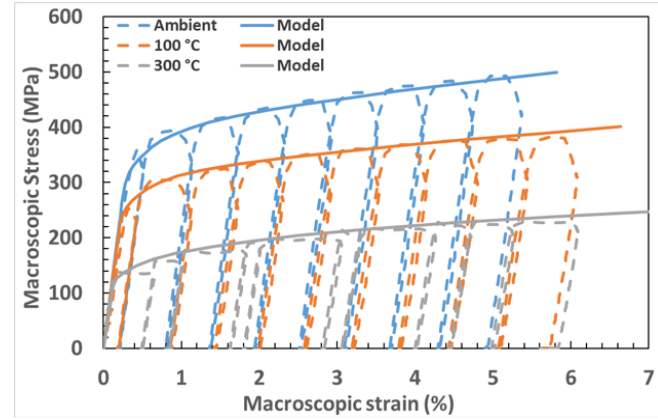

Fig. 3: Observed (dashed lines) and calculated (solid lines) macroscopic stress-strain curves at ambient, 100 and $300{ }^{\circ} \mathrm{C}$

\section{Discussion}

Fig. 4 shows the residual lattice strain development during the tensile tests, which are the elastic strains measured in grains oriented for diffraction after unloading. The // and $\perp$ symbols refer respectively to the lattice strain in the parallel (i.e. longitudinal) and perpendicular (i.e. transversal) directions to the stress axis (corresponding to the extrusion direction). The results clearly highlight an anisotropic mechanical behavior of \{hk.1\} groups of grains. As seen in Fig.4, after tensile loading, significant positive residual strains develop in the $\{00.2\} / /$ grains at ambient, 100 and $300{ }^{\circ} \mathrm{C}$ (respectively, 450,430 and $510 \mu \varepsilon$ ) at $5 \%$ of total strain. Contrary to \{00.2\}// grain familly, negative strains are observed for $\{10.0\}_{/ /}$and $\{10.1\}_{/ /}$reflections. Most of the residual lattice strains are accumulated within the first $1 \%$ of total strain.
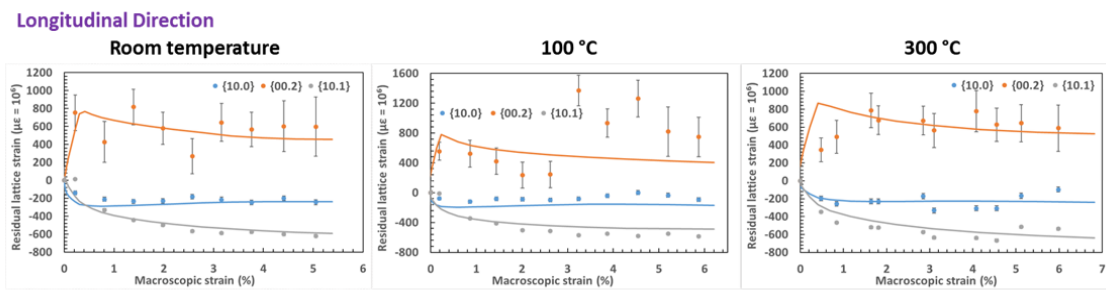

Transversal Direction
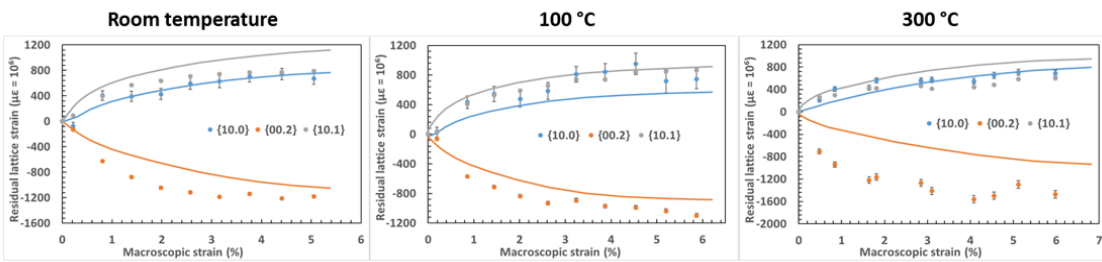

Fig. 4: Residual experimental and predicted lattice strain response in both longitudinal and transversal directions for axial tensile samples at ambient, 100 and $300{ }^{\circ} \mathrm{C}$

Residual strain evolution of the $\{\mathrm{hk} .1\}$ reflections can be correlated with the activation of particular deformation modes. Along the loading direction, the $\{10.0\} / /$ diffracting grains noticeably begin shedding load above 265,212 and $108 \mathrm{MPa}$ at ambient, 100 and $300{ }^{\circ} \mathrm{C}$, respectively. This can be rationalized by the activation of the prismatic slip (denoted pr $>$ in Fig. 5), which has the lowest CRSS (Table 1) combined with the highest Schmid factor for these orientations. It undergoes the plastic deformation below the macroscopic yield stress. $\{00.2\} / /$ planes develop significant tensile residual lattice strains $(\sim 800 \mu \varepsilon$ at room temperature $)$ during the first $1 \%$ of total strain. This indicates that the $\{00.2\} / /$ grains support a larger fraction of each stress increment after plasticity. This is expected since these grains are poorly orientated for the soft $>$ slip modes. The plasticity of $\{00.2\} / /$ diffracting grains corresponds to the activation of pyramidal $>$ system (denoted pyr $>$ ) for the three studied temperatures. 
Room temperature

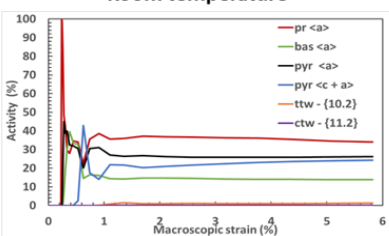

$100^{\circ} \mathrm{C}$

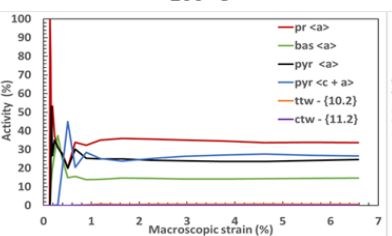

$300^{\circ} \mathrm{C}$

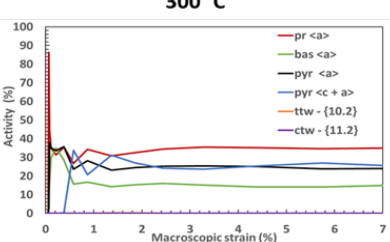

Fig. 5: Relative contribution of each deformation mode to the total shear as a function of deformation

Fig. 4 shows the measured and modeled lattice response in grains with \{hk.1\} plane-normal parallel and perpendicular to the applied stress in tension. The model predictions of the residual strains parallel to the tensile axis are fairly accurate for the studied reflections in term of trend and magnitude. The use of EPSC model allows to explain the origin of these residual strains.

As shown in Fig. 5, the main deformation mode for the different temperatures, among the various deformation mechanisms under tension is prismatic slip (pr $>$ ). Tensile $\{10.2\}\langle 10 . \overline{1}\rangle(\mathrm{ttw}-\{10.2\})$ and compressive ${ }^{\{11.2\}\langle 11 . \overline{3}\rangle}(\mathrm{ctw}-\{11.2\})$ twinning modes have the smallest predicted relative activities. In this way, the contribution of $\operatorname{ctw}-\{11.2\}$ system can be neglected. The activity of the twinning ttw- $\{10.2\}$ decreases as the temperature increases. This is consistent with previous works $[7,22,23]$.

\section{Conclusion}

The evolution of residual lattice strains and texture during the deformation of an extruded $\alpha$-Ti alloy have been examined using in situ neutron diffraction. These results are compared to an extended EPSC model taking into account twin domain reorientation and associated stress relaxation. On the basis of this study, the relative activity of slip and twinning deformation modes occurring at various temperatures have been determined and analyzed. By tuning the parameters of the EPSC model (hardening parameters and Critical Resolved Shear Stresses), it is shown that the model predictions in terms of lattice strain evolution and macroscopic stress-strain curves are in good agreement with the experimental data. Our work highlights the strong temperature effect onto the mechanical response of $\alpha$-Ti under uniaxial tension, notably the evolution of lattice strains, the roles of twin activity and the crystallographic texture.

\section{Acknowledgements}

The authors thank the ISIS Neutron Facilities scientific committees for the allocated experimental days on Engin-X and GEM (ISIS, UK) instruments. In particular, S. Kabra, J. Kelleher and W. Kockelmann are gratefully thanked for fruitful discussions and support.

\section{References}

[2] H. Sibum, Adv. Eng. Mater., 5, (2003) 393-398.

[3] P. G. Partridge, Metall. Rev., 118, (1967) 169-194.

[4] M. G. Glavicic, A. A. Salem, and S. L. Semiatin, Acta Mater., 52, (2004) 647-655.

[5] A. A. Salem, S. R. Kalidindi, and R. D. Doherty, Acta Mater, 51, (2003) 4225-4237.

[6] D. R. Chichili, K. T. Ramesh, and K. J. Hemker, Acta Mater., 46, (1998) 1025-1043.

[7] H. Conrad, Prog. Mater. Sci., 26, (1981) 123-403.

[8] H. R. Wenk, S. Matthies, J. Donovan, and D. Chateigner, J. Appl. Crystallogr., 31, (1998) 262-269. 
[9] A. C. Hannon, Nucl. Instruments Methods Phys. Res. A, 551, (2005) 88-107.

[10] J. R. Santisteban, M. R. Daymond, J. A. James, and L. Edwards, J. Appl. Crystallogr., 39, (2006) 812-825.

[11] C. M. Moreton-Smith, S. D. Johnston, and F. A. Akeroyd, J. Neutron Res., 4, (1996) 41-47.

[12] D. Gloaguen, G. Oum, V. Legrand, J. Fajoui, and S. Branchu, Acta Mater., 61, (2013) 5779-5790.

[13] D. Gloaguen, T. Berchi, E. Girard, and R. Guillén, Acta Mater, 55, (2007) 4369-4379.

[14] P. Lipinski and M. Berveiller, Int. J. Plast., 5, (1989) 149-172.

[15] B. Clausen, C. N. Tomé, D. W. Brown, and S. R. Agnew, Acta Mater., 56, (2008) 2456-2468.

[16] G. Simmons and H. Wang, The MIT Press. 1971.

[17] P. A. Turner, N. Christodoulou, and C. N. Tomé, Int. J. Plast., 11, (1995) 251-265.

[18] D. Rodríguez-Galán, I. Sabirov, and J. Segurado, Int. J. Plast., 70, (2015) 191-205.

[19] E. S. Fisher, J. Nucl. Mater., 18, (1966) 39-54.

[20] B. Barkia, V. Doquet, J. P. Couzinié, I. Guillot, and E. Héripré, Mater. Sci. Eng. A, 636, (2015) 91-102.

[21] B. Barkia, V. Doquet, J. P. Couzinié, I. Guillot, and E. Héripré, Mater. Sci. Eng. A, 636, (2015) 91-102.

[22] N. E. Paton and W. A. Backofen, Metall. Trans., 1, (1970) 2839-2847.

[23] R. Sánchez-Martín, C. Zambaldi, M. T. Pérez-Prado, and J. M. Molina-Aldareguia, Scr. Mater., 104, (2015) 9-12. 